\title{
La producción científica de la revista Acta Colombiana de Psicología: Análisis descriptivo y bibliométrico del periodo 2015-2019
}

\author{
Ernesto L. Ravelo-Contreras; Miguel Barboza-Palomino; Susana Mejía; Juan David Millán; Gonzalo Salas
}

How to cite this article:

Ravelo-Contreras, E.L., Barboza-Palomino, M., Mejía, S., Millán, J.D., \& Salas, G. (2020). The scientific production of Acta Colombiana de Psicología: descriptive and bibliometric analysis of the period $2015-2019$. Acta Colombiana de Psicología, 23(2), 170-187. http://www.doi.org/10.14718/ACP.2020.23.2.8

Recibido, noviembre 20/2019; Concepto de evaluación, enero 07/2020; Aceptado, enero 16/2020

\author{
Ernesto L. Ravelo-Contreras* \\ Universidad Católica de Colombia, Bogotá, Colombia \\ ORCID: https://orcid.org/0000-0002-9565-0828 \\ Miguel Barboza-Palomino \\ Universidad Privada del Norte, Trujillo, Perú \\ ORCID: https://orcid.org/0000-0001-8045-5491 \\ Susana Mejía \\ Universidad Católica de Colombia, Bogotá, Colombia \\ ORCID: https://orcid.org/0000-0002-1866-672X \\ Juan David Millán \\ Universidad Católica del Maule, Talca, Chile \\ ORCID: https://orcid.org/0000-0001-9652-7664 \\ Gonzalo Salas \\ Universidad Católica del Maule, Talca, Chile \\ ORCID: https://orcid.org/0000-0003-0707-8188
}

\begin{abstract}
Resumen
El presente artículo tuvo como objetivo la descripción y el análisis bibliométrico de los artículos publicados en la revista Acta Colombiana de Psicología entre los años 2015 y 2019. Se analizó su evolución y tendencias con el fin de implementar estrategias que permitan la permanente cualificación de la revista, sobre todo en cuanto a calidad científica, visibilidad, impacto y cobertura geográfica. Para esto, se construyó una base de datos con 127 artículos científicos, con los cuales se realizaron análisis descriptivos — frecuencias y porcentajes - y se calcularon algunos índices bibliométricos — como el índice de productividad, los índices de Lawani y Subramanyam, y el índice de Pratt- mediante una planilla en Excel complementada con el paquete estadístico IBM SPSS, versión 23. Los hallazgos más significativos permiten observar un importante número de trabajos con autoría internacional (74\%), un desarrollo elevado de artículos en el área de psicología clínica y de la salud (42.5\%), así como en psicometría (21.3\%), y un énfasis de trabajos de corte cuantitativo (87\%). La revista continúa realizando aportes significativos para el desarrollo de la psicología en Hispanoamérica.

Palabras clave: Acta Colombiana de Psicología, bibliometría, indicadores, producción científica, revista, psicología, coautoría.
\end{abstract}

* Calle 66 No. 59-31 Torre 9 Apartamento 407 Bogotá, D.C., Colombia. Tel.: (57-1) 7035354, ravelocontreras@gmail.com 


\title{
The scientific production of Acta Colombiana de Psicología: descriptive and bibliometric analysis of the period 2015 - 2019
}

Abstract

\begin{abstract}
The objective of this article is the description and bibliometric analysis of the articles published in the journal Acta Colombiana de Psicología between 2015 and 2019. Their evolution and trends are analyzed to implement strategies that allow their permanent qualification regarding a) scientific quality, b) visibility, c) impact and d) geographic coverage, among others. The database included 127 scientific articles. Descriptive analyzes (frequencies and percentages) were performed and some bibliometric indices were calculated: a) productivity index, b) Lawani and Subramanyam indices and c) Pratt index. These analyzes were carried out using an Excel spreadsheet supplemented with the IBM SPSS version 23 statistical package. The most significant findings allow observing an important number of papers with international authorship (74\%), high development of articles in the areas of clinical and health psychology (42.5\%) and psychometry $(21.3 \%)$ and an emphasis on works of a quantitative nature (87\%). The journal continues making significant contributions to the development of psychology in Latin America.

Keywords: Acta Colombiana de Psicología, bibliometrics, indicators, scientific production, journal, psychology, co-authorship.
\end{abstract}

\section{Introducción}

Los estudios sobre la evaluación de la productividad de una ciencia a partir de sus publicaciones en revistas científicas son cada vez más usuales y pertinentes para el avance disciplinar y profesional (Aguado-López et al., 2017; Fierro et al., 2017; Morgado-Gallardo et al., 2018; Puche-Navarro \& Ossa, 2012), a la vez que han sido considerados el primer paso para analizar las dinámicas de una comunidad académica (Vinkler, 2010). Robert Merton, el sociólogo norteamericano, pensaba que una buena práctica científica consiste en compartir los resultados con otros, de modo que el progreso científico pueda medirse por el crecimiento del número de autores, citaciones y publicaciones, entre otras variables asociadas (Daniel, 2005; Millán et al., 2017). Así, la ciencia, según la bibliometría, se comporta como un sistema de comunicaciones compuesto por autores, artículos y discursos conectados por un código semántico determinado (Leydesdorff, 2007).

Ahora bien, cada vez que se emprende el desafío de realizar una bibliometría sobre una revista científica de un área particular, es importante tener cuidado de no caer en una suerte de empresa descriptiva y celebratoria de la revista, $o$, en otras palabras, convertir el estudio bibliométrico en un simple homenaje (ver Kamdem et al., 2019; Milfont et al., 2019; Sigué, 2019; Wang et al., 2019). Por el contrario, como en algunos estudios bibliométricos realizados respecto a la producción académica en revistas científicas de psicología latinoamericanas, lo más importante es poner en evidencia tanto la situación de la disciplina como sus respectivos problemas y desafíos nacionales y regionales (Cudina et al., 2017; Gómez-Morales et al., 2012; Jaraba-Barrios et al., 2012; López-López \& Calvache, 1998; Salas et al., 2017).
Adicional a ello, la utilización de las revistas en dichos estudios también permite dar cuenta del progreso de la actividad científica, así como algunos asuntos estructurales de ella, como los efectos de las políticas nacionales de incentivo científico, la infraestructura editorial o fenómenos derivados de la cada vez más convulsa actividad científica (Calver et al., 2013; Huang et al., 2014).

Teniendo lo anterior en cuenta, el presente estudio continúa la senda de dos trabajos previos vinculados a la revista Acta Colombiana de Psicología [de aquí en adelante, Acta] —el de Ravelo-Contreras et al. (2016;), y el de Salas et al. (2018) - , con los cuales es posible realizar un análisis descriptivo y bibliométrico de la revista para estudiar su evolución, sus tendencias y la implementación de estrategias para su continua cualificación, sobre todo en cuanto a su calidad, visibilidad, impacto y cobertura geográfica, entre otros.

Acta, desde su fundación en 1998 en la Facultad de Psicología de la Universidad Católica de Colombia, se ha convertido no solo en una plataforma de divulgación, sino también en un "indicador" que ha permitido trazar una parte importante de la investigación psicológica en Colombia y Latinoamérica, tal como se ha evidenciado en estudios bibliométricos e históricos realizados con anterioridad (Ardila, 1999; Ravelo-Contreras et al., 2016; Salas et al., 2018; Salas et al., 2019).

En sus inicios, la revista empezó su publicación con un número anual hasta el año 2000, para, a partir de ese momento, publicar sus números de forma semestral; esto en consecuencia con lo establecido en la primera editorial a cargo del profesor Carlos Vargas Ordoñez — quien se desempeñaba como decano de la Facultad de Psicología de la Universidad Católica de Colombia—, donde se trazó el proyecto editorial de la revista, en el cual se dejó en claro 
172

que la misma estaría dedicada tanto al avance conceptual como a la aplicación práctica de la psicología, en especial de los campos clínico y educativo, al igual que en la medición psicométrica (Vargas-Ordoñez, 1998).

En términos históricos, la apertura del Centro de Estudios e Investigaciones sobre las Adicciones (CEIA-UCC) en la Facultad de Psicología en 1997 tuvo una gran incidencia en el temario inicial de los artículos publicados en el primer y cuarto número, con un fuerte énfasis en la farmacodependencia y el tratamiento de las adicciones (Flórez-Alarcón, 1998, 2000). Posteriormente, gracias a las profesoras Beatriz Molano García y Martha Cecilia Lozano Ardila, quienes participaron con el rol de editoras invitadas, la psicología educativa fue el tema principal de la revista, especialmente en el segundo y tercer número - siguiendo la modalidad de números especiales - (Lozano-Ardila, 2000; MolanoGarcía, 1999). Después, el quinto número fue dedicado a los estudios psicométricos, mientras que, como consecuencia de la apertura del posgrado en derecho, los artículos del sexto número abordaron el tema de la psicología jurídica y su interdisciplinariedad con dicha disciplina (VargasEspinosa, 2001). Después de esto, en el décimo número se buscó el avance de la psicología básica, sobre todo gracias a los estudios de varios investigadores nacionales y de la misma facultad en este tema (Cruz-Becerra, 2003); y luego, también por motivos del contexto en el que se encontraba la universidad en esos momentos, los números undécimo y duodécimo estuvieron dirigidos a la psicología organizacional y social.

En esta época, y a partir de ella, es importante destacar que el trabajo editorial y científico de Acta ha estado también íntimamente relacionado con el desarrollo de campos profesionales no tan usuales en la psicología en Colombia — los denominados "áreas con menor porcentaje de profesionales" - , además de que ha sido un medio de internacionalización de la psicología colombiana de gran relevancia, pues fue una de las primeras revistas del país en ser indexada en bases de datos internacionales como PSICODOC — del Colegio de Psicólogos de Madrid—, y en ser rápidamente sometida a evaluación para su inclusión en PsycINFO - de la American Psychological Association(Ardila \& Pérez-Acosta, 2010; Pérez-Acosta, 2002).

Posteriormente, en el número 13 de la revista se publicaron artículos relacionados con investigaciones de psicología clínica con énfasis cognoscitivo y conductual, modelo epistemológico que tomaba fuerza en la Especialización

\footnotetext{
${ }^{1}$ Rubén Ardila encontró en 1999 que las áreas con menor porcentaje de profesionales son la psicología deportiva, la psicología de la salud, psicología jurídica y criminológica, la neuropsicología y la psicología gerontológica (Ardila 1999).
}

y en la Maestría en Psicología Clínica de la Universidad Católica de Colombia (Flores-Alarcón, 2005). Después, entre 2005 y 2008, Acta tuvo importantes avances, como su indexación en bases de datos internacionales como Scopus, Redalyc, Dialnet, DOAJ, Ulrich, IRESE (RaveloContreras, 2007; Solís-Camara \& Díaz-Romero, 2007), y en la diversificación de los temas en sus publicaciones, pues, como reconoció Rubén Ardila en 2006: "se hace cada vez más usual la publicación de artículos en las líneas de la psicología de la salud, asuntos educativos desde dos perspectivas diferentes, psicología deportiva, psicología de la paz y de la violencia, psicología del trabajo y de las organizaciones, incluyendo selección de personal" (p. 4). En este momento, cabe destacar que la revista adoptó la publicación por medio del formato de "volumen" y "número", siendo el volumen 9 número 1 el primero publicado bajo esa modalidad.

Después, en 2008 Acta se categorizó "A1" en el sistema Publindex, y a partir de ese mismo año se inició un periodo fructífero en materia de calidad científica y de consolidación de redes de cooperación con revistas de la región, como la Revista Mexicana de la Conducta y la Revista Latinoamericana de Medicina Conductual (RaveloContreras, 2008, 2012). Así, en el periodo de 2008 a 2014 destacan el volumen 16, número 2 —un número especial a cargo de la Doctora María del Rocío Hernández-Pozo (2013) — dedicado a difundir investigaciones latinoamericanas sobre equidad de género desde diferentes perspectivas y enfoques metodológicos; y el volumen 17, número 2, del 2014, el cual contribuyó a la divulgación de investigaciones relacionadas con las áreas de psicofisiología, psicobiología y neuropsicología en cualquiera de sus campos de intervención (clínico, educativo y básico) (Salvador-Cruz, 2014). Aquí, cabe señalar que a pesar de que la revista abandonó la publicación de números especiales, todos sus artículos publicados cuentan con la participación de autores de diversas instituciones y están orientados a la investigación interdisciplinaria y contextualizada de problemáticas que aparecen como consecuencia de los cambios en la sociedad y su impacto en el individuo, la familia, la cultura, el colectivo, etc.

Finalmente, como consecuencia de las exigencias de algunas bases e índices de datos internacionales, además de la necesidad de obtener mayor visibilidad en comunidades académicas, y con el objetivo de aumentar la cobertura geográfica de los lectores, desde el año 2016 los artículos publicados fueron traducidos al inglés y al portugués (Ravelo-Contreras, 2016). En ese mismo año, gracias al apoyo de su institución editora, Acta fue clasificada en la categoría Q3 en el Ranking Scimago y ubicada en la categoría B en Publindex, ambos hechos ponderables para la 
Tabla 1.

Ejes temáticos de los números publicados en el periodo entre 2015 y 2019

\begin{tabular}{|c|c|c|}
\hline Año & Volumen y número & Ejes temáticos \\
\hline 2015 & Vol. 18, núm. 1 & $\begin{array}{c}\text { Artículos publicados gracias a la contribución de la Red Nacional de } \\
\text { Psicología Experimental humana (RMIPEH), del Sistema Mexicano de Inves- } \\
\text { tigación en Psicología (SMIP). }\end{array}$ \\
\hline 2015 & Vol. 18, núm. 2 & $\begin{array}{c}\text { Artículos publicados gracias a la contribución de la Red Mexicana de } \\
\text { Investigación en Psicología Educativa, del Sistema Mexicano de Investigación } \\
\text { en Psicología (SMIP). }\end{array}$ \\
\hline 2016 & Vol. 19, núm. 1 & $\begin{array}{c}\text { Psicometría, psicología social y organizacional, estudios teóricos sobre } \\
\text { psicología educativa. }\end{array}$ \\
\hline 2016 & Vol. 19, núm. 2 & Psicometría, neuropsicología y violencia de género. \\
\hline 2017 & Vol. 20, núm. 1 & Salud mental, psicometría, bullying y programas de intervención psicológica. \\
\hline 2017 & Vol. 20, núm. 2 & $\begin{array}{l}\text { Psicología clínica, burnout, estudios de la personalidad, y estudios sobre } \\
\text { familia. }\end{array}$ \\
\hline 2018 & Vol. 21, núm. 1 & Cyberbullying y escalas de medición. \\
\hline 2018 & Vol. 21, núm. 2 & Bibliometría, actitudes políticas en jóvenes, e inteligencia emocional. \\
\hline 2019 & Vol. 22, núm. 1 & Psicometría, psicología de la salud, y psicología comunitaria aplicada. \\
\hline 2019 & Vol. 22, núm. 2 & $\begin{array}{c}\text { Psicología de la salud, neuropsicología, psicología educativa, psicología } \\
\text { básica, psicometría y psicología de la percepción. }\end{array}$ \\
\hline
\end{tabular}

Nota. Fuente: elaboración propia.

historia de las publicaciones de la psicología en Colombia (Ravelo-Contreras, 2017). Por último, en el año 2019 Acta cumplió 22 años de publicación ininterrumpida, y se ha transformado en un medio de conformación de redes nacionales e internacionales.

El presente artículo de investigación se centra en los últimos cinco años de la revista, es decir, los comprendidos en el período entre 2015 y 2019, en los cuales Acta ha publicado diez números que abarcan temas que van desde la psicología experimental hasta la psicología de la percepción (véase Tabla 1). Este estudio evidencia de manera objetiva, y a través de indicadores y técnicas bibliométricas el último quinquenio de la revista.

\section{Método}

\section{Tipo de estudio}

Se realizó un estudio ex post facto retrospectivo de tipo bibliométrico y de carácter descriptivo a partir de los artículos publicados por una revista científica (Montero \& León, 2007; Utap-Anyi et al., 2009), donde se utilizaron los siguientes indicadores: (a) el número total de artículos, (b) el sexo de los autores, (c) el idioma de la publicación, (d) el índice de productividad, (e) los índices de colaboración - Subramanyam y Lawani-, (f) el índice de medición de concentración temática —Pratt—, (g) el país de afiliación, (h) las áreas temáticas, y, por último, (i) las metodologías y diseños implementados en cada artículo (Alhuay-Quispe \& Pacheco-Mendoza, 2018; Egghe, 1988; Lawani, 1981, 1986; Pratt, 1977; Subramanyan, 1983; Travis-Nichols, 1989).

\section{Universo del estudio}

Se analizó un total de 127 artículos publicados en el periodo comprendido entre el año 2015 y el año 2019. Para el análisis, se incluyeron solo los artículos científicos y se excluyeron las editoriales, la información institucional y las reseñas.

\section{Instrumentos}

Se construyó una planilla en Excel alimentada con todos los documentos de la revista con el fin de obtener la base de datos que sería la fuente de análisis de los métodos bibliométricos utilizados. Esta base de datos fue creada con la información del sitio web oficial de la revista (Universidad Católica de Colombia, 2015-2019).

\section{Procedimiento}

Una vez constituido el corpus empírico, se procedió a organizar la información en una base de datos para hacer el análisis bibliométrico y descriptivo a partir de indicadores clásicos bibliométricos y algunas métricas habituales en los estudios sobre la producción académica de una revista (Bonnevie, 2002; Lievrouw, 1989; Tsay, 2003; Tsay et al., 2000). 
174

Análisis de datos

La minería del corpus empírico se llevó a cabo a partir de la información obtenida en la página web de la revista, la cual fue verificada con la base de datos de Scopus. Posteriormente, se calcularon tanto los indicadores descriptivos como los índices de productividad, de colaboración y de concentración temática, con apoyo de una hoja de cálculo en el programa Excel y el paquete estadístico IBM SPSS, versión 23.

\section{Resultados}

Para responder al objetivo del estudio, se realizaron los análisis descriptivos -frecuencias y porcentajes - para cada una de las variables categóricas. En cuanto a las variables cuantitativas, se hizo uso de la media y la desviación estándar. Finalmente, se hizo el respectivo cálculo de los índices bibliométricos descritos en la sección del método del estudio.

\section{Resultados descriptivos por variable}

Análisis de variables categóricas o cualitativas

Entre los años 2015 y 2019, la revista Acta Colombiana de Psicología ha publicado 127 artículos, es decir, entre 24 y 29 artículos por año, con una periodicidad de publicación semestral (véase Tabla 2). Como se muestra en la Figura 1, el $74.9 \%$ de los autores que han publicado durante este periodo corresponden a investigadores internacionales, el $11 \%$ son autores nacionales externos a la Universidad Católica de Colombia, y solo el $4.7 \%$ de los trabajos son realizados por docentes de la institución.
Tabla 2.

Número de publicaciones por año

\begin{tabular}{cc}
\hline Año & N (\%) \\
\hline 2015 & $29(22.83)$ \\
2016 & $24(18.9)$ \\
2017 & $24(18.9)$ \\
2018 & $24(18.9)$ \\
2019 & $26(20.47)$ \\
\hline Total & 127 \\
\hline
\end{tabular}

En cuanto al idioma, el $77 \%$ de los artículos se escribieron originalmente en idioma español, el $14 \%$ en portugués y el $9 \%$ en inglés (véase Figura 2). Sin embargo, a partir del año 2016, todos los artículos se han publicado tanto en inglés como en español. Por otra parte, con respecto al área de conocimiento de la revista, 54 artículos (el $42.5 \%$ del total) corresponden al área de psicología clínica o de la salud, 27 artículos (21.3\%) fueron de psicometría, 15 (11.8 \%) fueron de psicología educativa, y 14 (11\%) hicieron aportes al área de la neuropsicología (véase Figura 3).

Específicamente, con respecto al tipo de investigaciones publicadas, el $87 \%$ corresponden a estudios cuantitativos, el $6 \%$ a estudios cualitativos y el $7 \%$ a metodologías mixtas (véase Figura 4); en cuanto al tipo de muestreo, en el $60.2 \%$ de los artículos que lo reportan se aplicó un muestreo no probabilístico, y en el $9.8 \%$, un muestreo probabilístico (véase Figura 5); y en relación con los instrumentos utilizados, en el $96.1 \%$ de los artículos se hizo uso de instrumentos para la recolección de la información, en el $9.4 \%$ se usaron encuestas y en el $2.4 \%$ se aplicaron entrevistas (véase Figura 6).

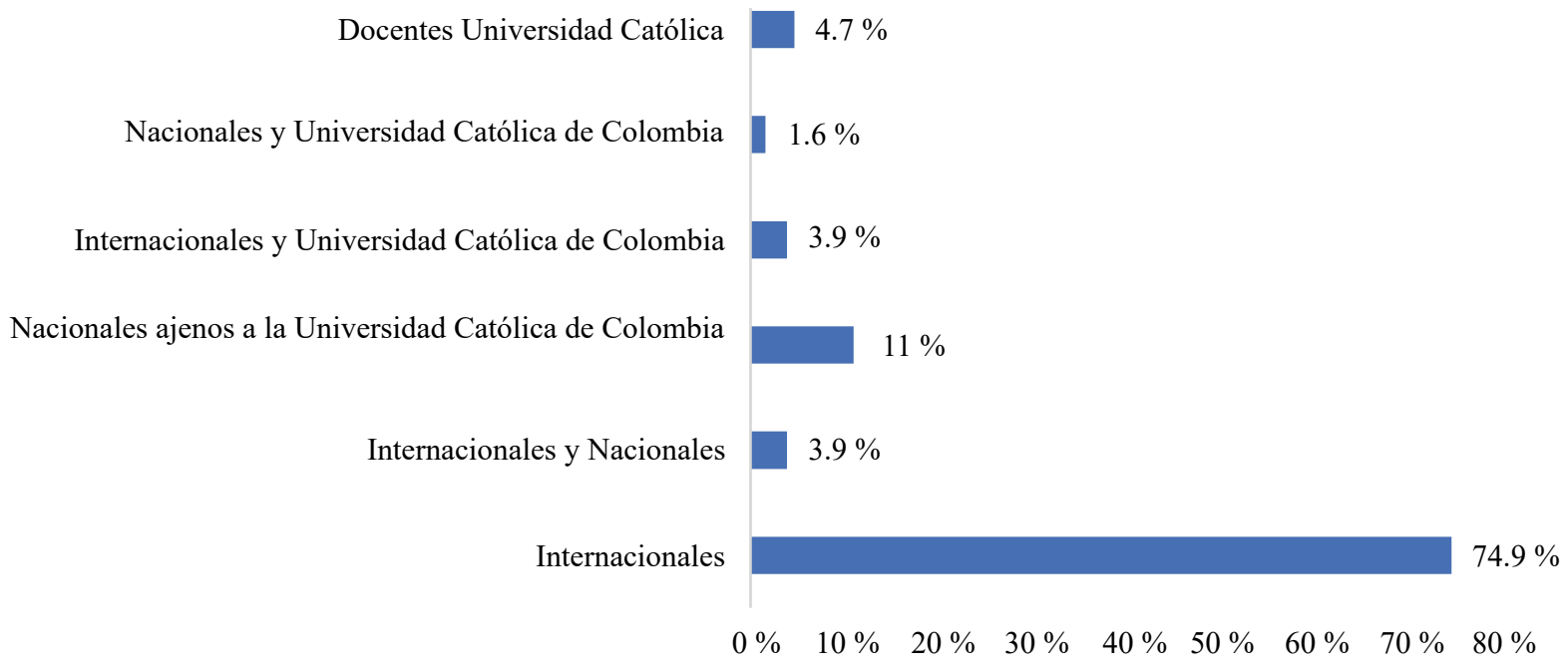

Figura 1. Procedencia institucional de los autores. 


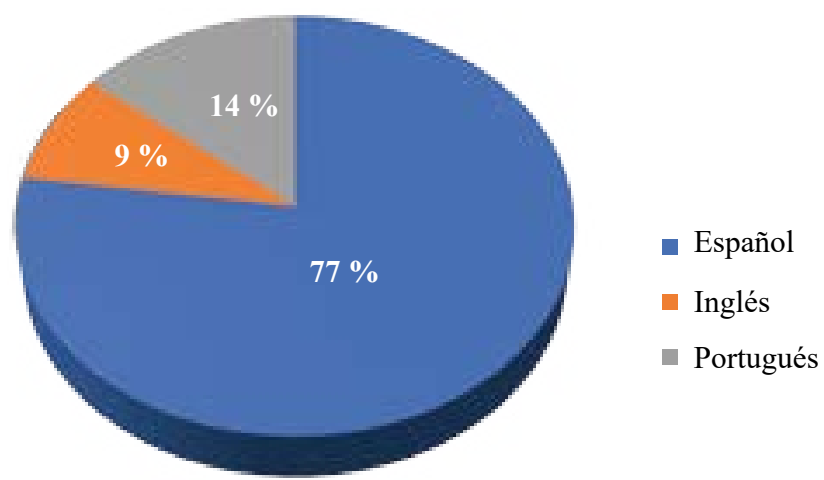

Figura 2. Porcentaje de idioma original.

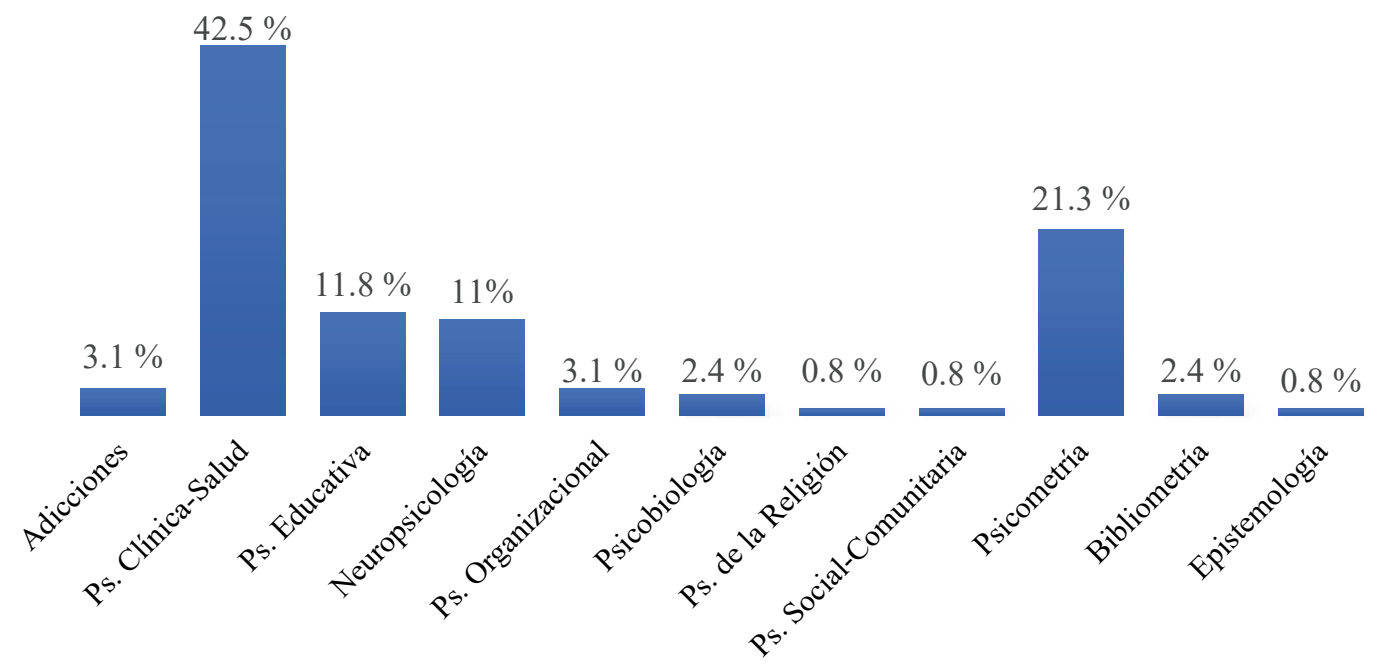

Figura 3. Porcentaje de publicaciones por área de conocimiento.

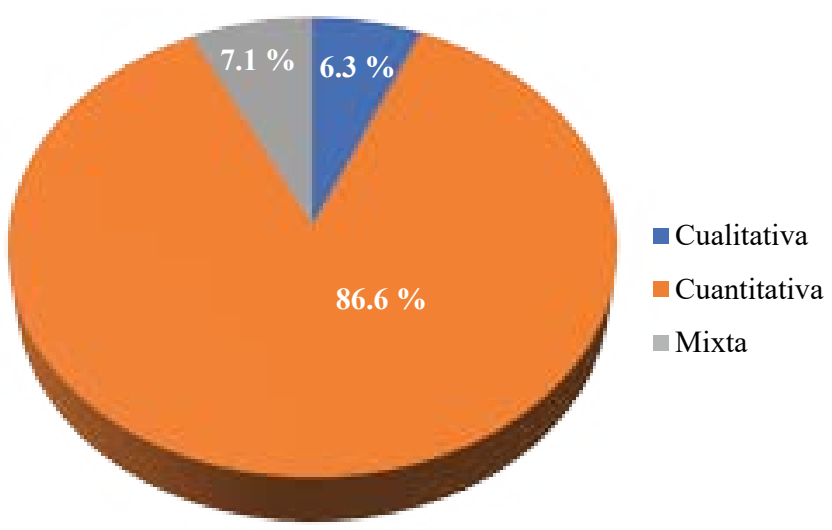

Figura 4. Publicaciones según el tipo de investigación. 
176

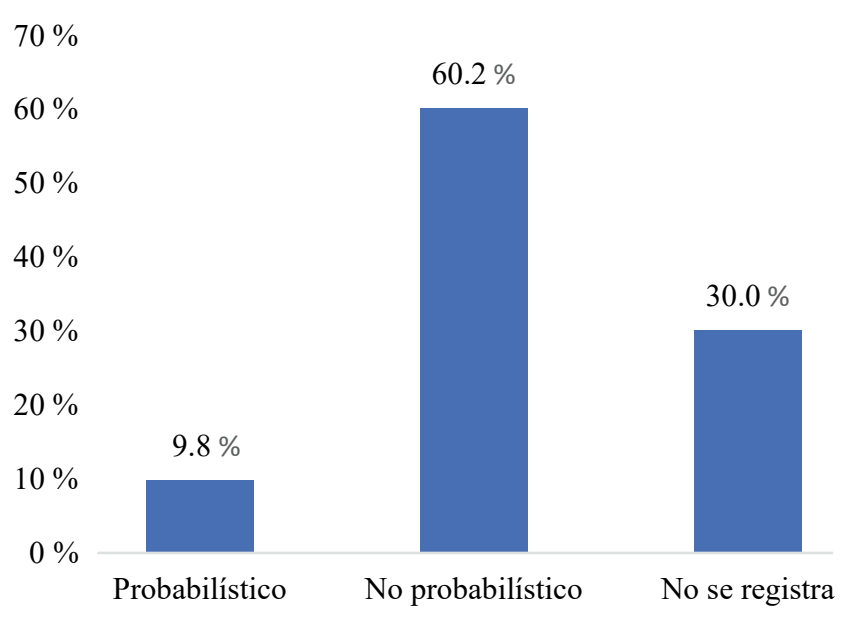

Figura 5. Publicaciones según el tipo de muestreo.

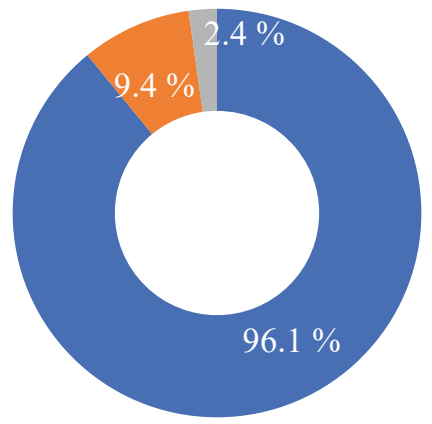

- Instrumento

Encuesta

$\square$ Entrevista

Figura 6. Publicaciones según el uso de instrumentos.

Por otro lado, se encontró que en el $97.6 \%$ de los estudios (124 artículos) se llevaron a cabo análisis descriptivos, en el $76.4 \%$ se hicieron análisis correlacionales, en el $53.5 \%$ se realizaron pruebas paramétricas, y en el $40.2 \%$ se hizo uso de pruebas no paramétricas, mientras que solo en el $29.9 \%$ de los estudios se emplearon los análisis multivariados (véase Figura 7).

\section{Análisis de variables cuantitativas}

Con respecto a los resultados del análisis de las variables cuantitativas, a continuación se presentan en cada caso el promedio y la desviación estándar (véase Tabla 3). Por ejemplo, el promedio del número de autores por artículo está entre 3 y 4; la diferencia entre el tiempo de recibido el artículo y el concepto frente al mismo se encuentra en un promedio de 5.17 ( \pm 3.87$)$ meses; mientras que el promedio entre el concepto y la aceptación es de $2.88( \pm 3.41)$ meses.

Tabla 3.

Estadísticos descriptivos

\begin{tabular}{ccc}
\hline & $M$ & $D E$ \\
\hline Número de autores & 3.32 & 1.57 \\
Tiempo recibido-concepto (meses) & 5.17 & 3.87 \\
Tiempo recibido-aceptado (meses) & 8.07 & 5.31 \\
Tiempo concepto-aceptación (meses) & 2.88 & 3.41 \\
Número de referencias & 45.02 & 16.60 \\
\hline
\end{tabular}

\section{Cruce de variables}

\section{Cruces por años}

Una vez realizados los análisis descriptivos para cada una de las variables se llevó a cabo la comparación por años. De este modo, como se puede observar en los datos registrados en la Figura 8, en la revista ha predominado el envío de artículos por parte de los autores en español, siendo este su idioma original, aunque se destaca que en el año 2015 el $17.2 \%$ de los artículos se publicaron en portugués. Por otra parte, se evidencia que en todos los años predominan las publicaciones de autores internacionales en todos los años, seguido por autores colombianos ajenos a la Universidad Católica de Colombia (véase Figura 9); y que, como se encuentra en la Figura 10 -donde se muestra el número de publicaciones por año según el área de conocimiento - en el año 2019 hubo un incremento importante en los artículos psicométricos.

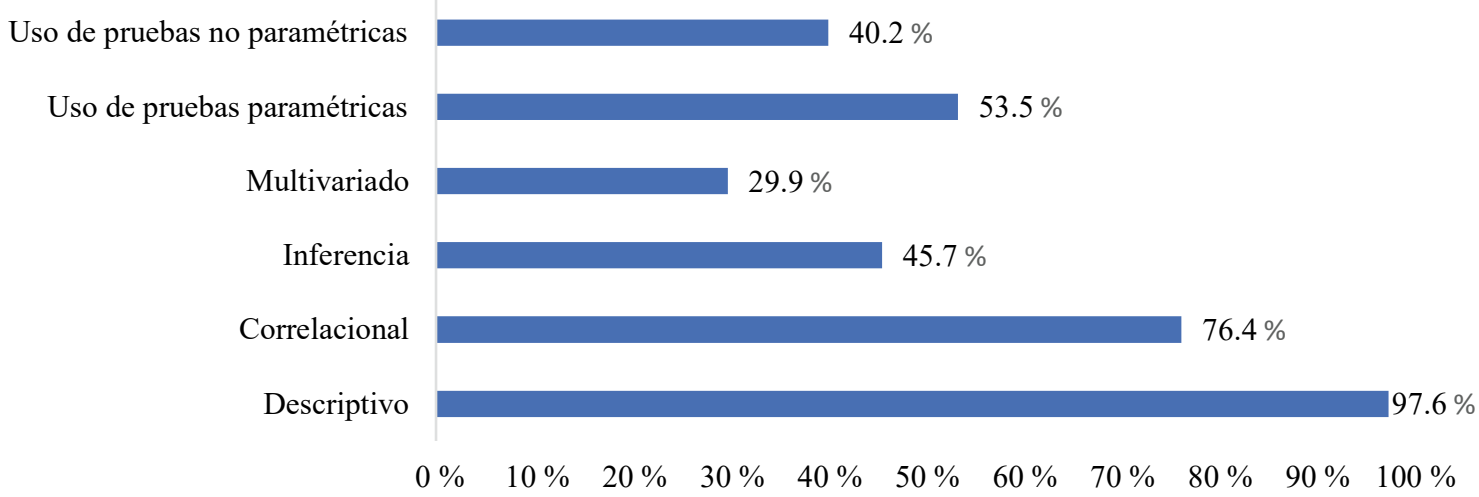

Figura 7. Publicaciones según el tipo de análisis estadístico. 


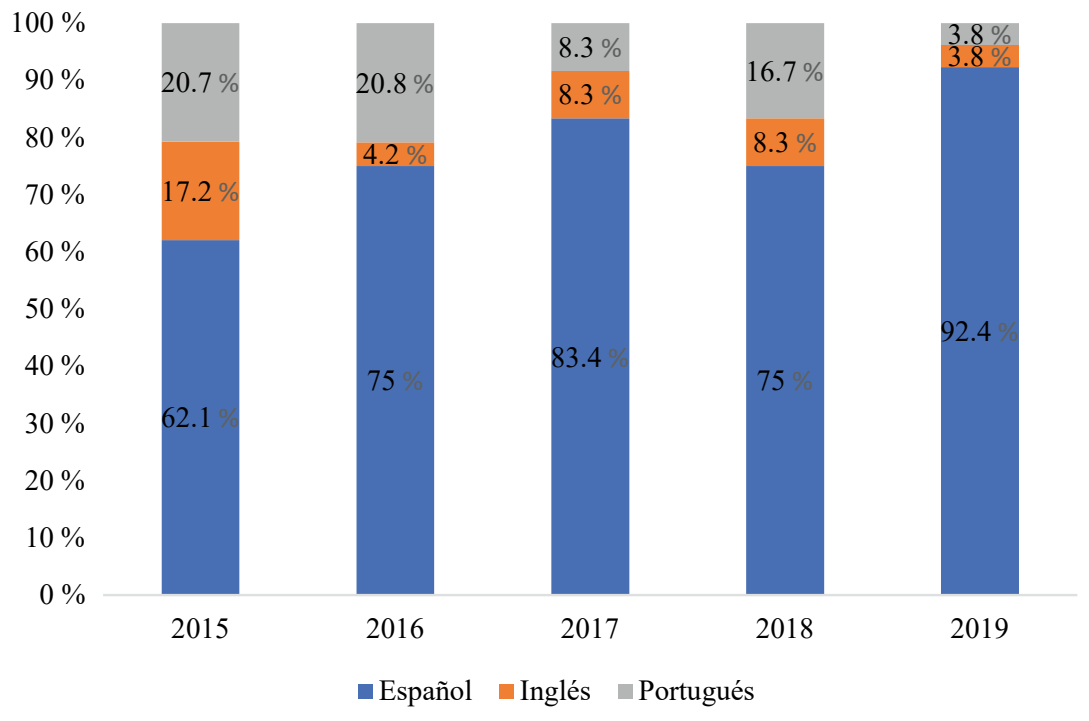

Figura 8. Idioma de publicación por año.

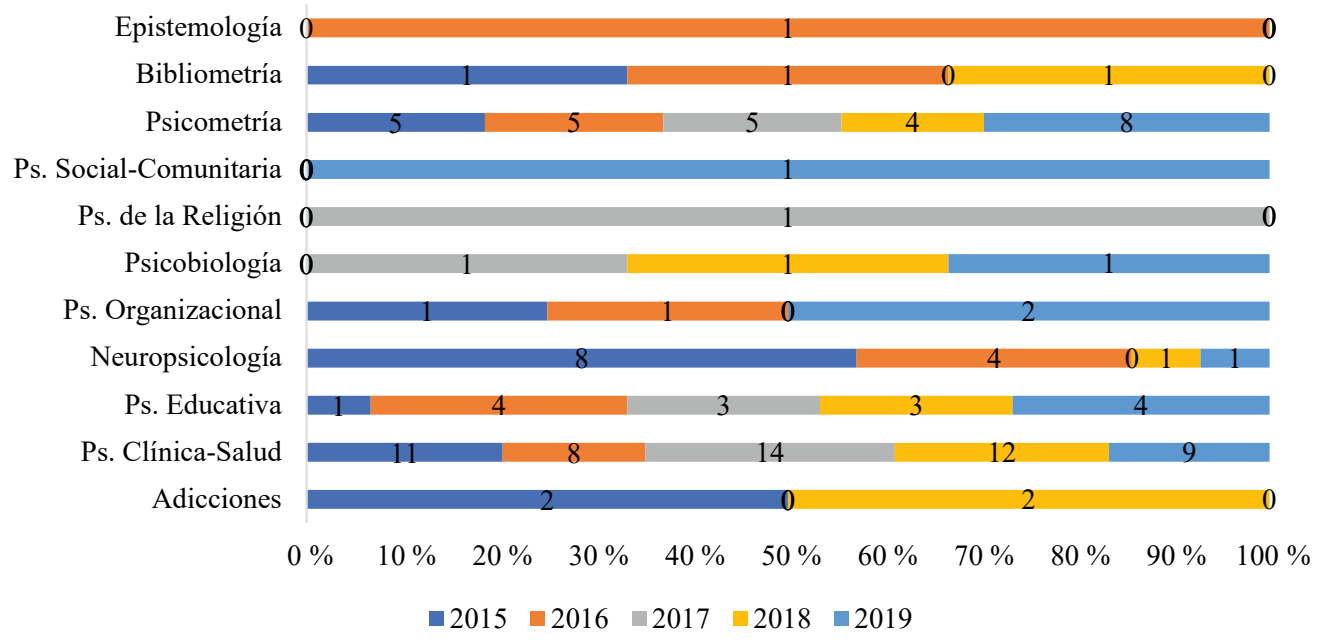

Figura 9. Procedencia de los autores, por año.

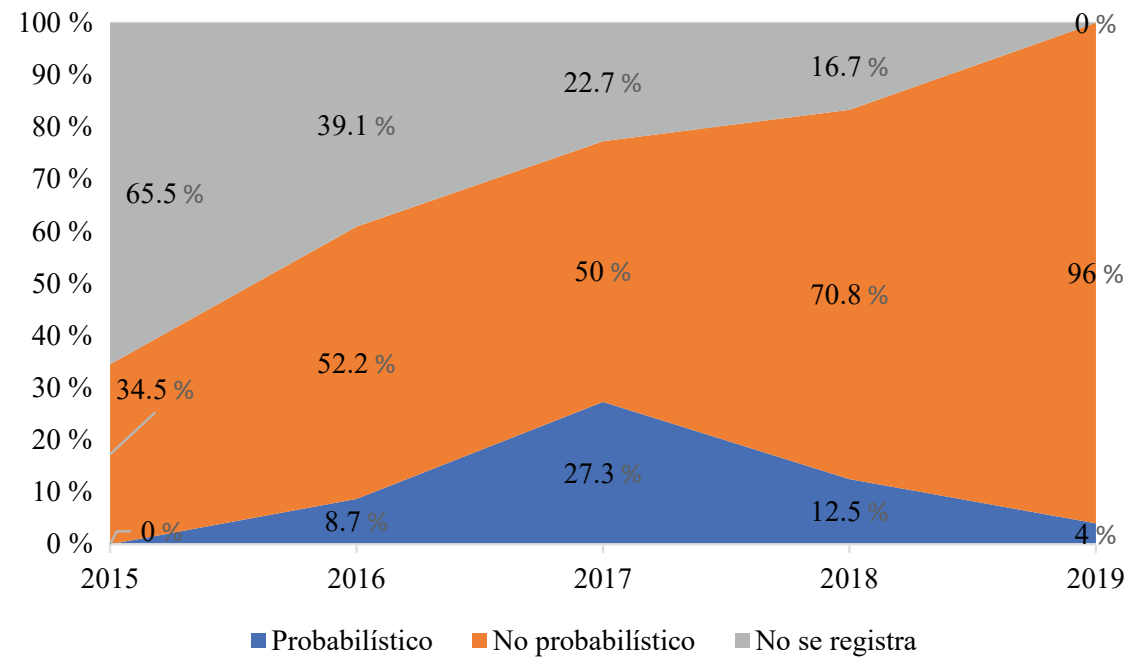

Figura 10. Publicaciones por año según el área de conocimiento. 
Por otra parte, en cuanto al tipo de muestreo, predomina el uso del muestreo no probabilístico (véase Figura 11), y llama la atención que a lo largo de los años se ha reducido el porcentaje de artículos en los que no se registra el tipo de muestreo empleado; incluso, en el año 2019 se reporta en el $100 \%$ de los estudios. Asimismo, predomina la investigación cuantitativa, con porcentajes que van entre el $54.2 \%$ y el
$100 \%$ por año (véase Figura 12); en más del $90 \%$ de las investigaciones se hizo uso del análisis descriptivos; y el empleo de análisis correlaciónales se evidencia en más del $65 \%$, mientras que los análisis inferenciales incrementaron su uso en el año 2019 —en el último año se redujo el uso de pruebas no paramétricas- (véase Figura 13).

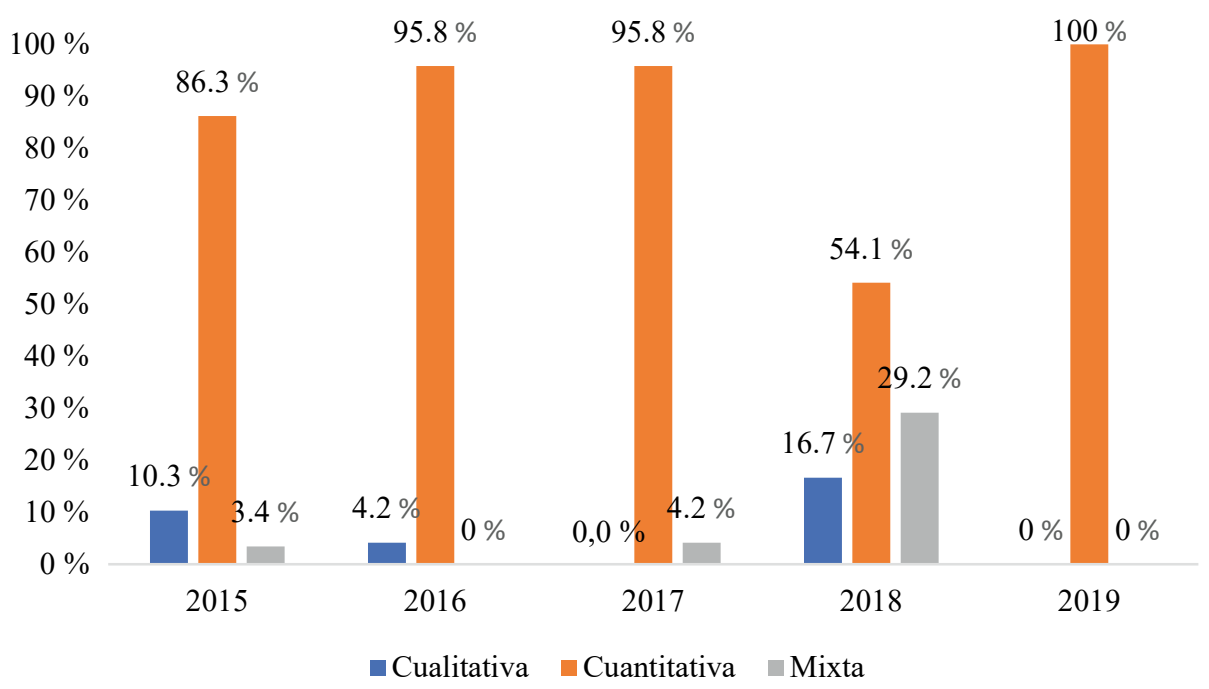

Figura 11. Publicaciones anuales según el tipo de muestreo.

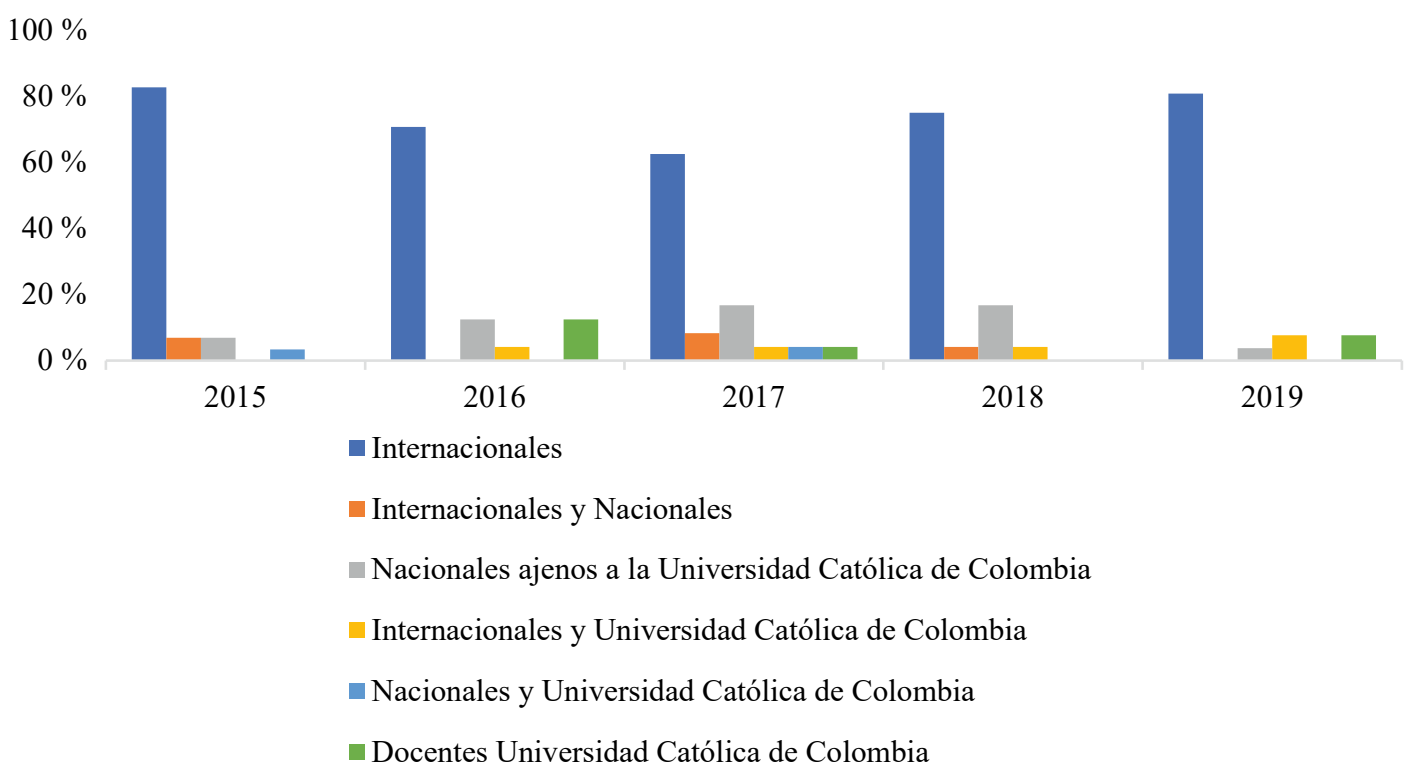

Figura 12. Publicaciones anuales según el tipo de investigación. 


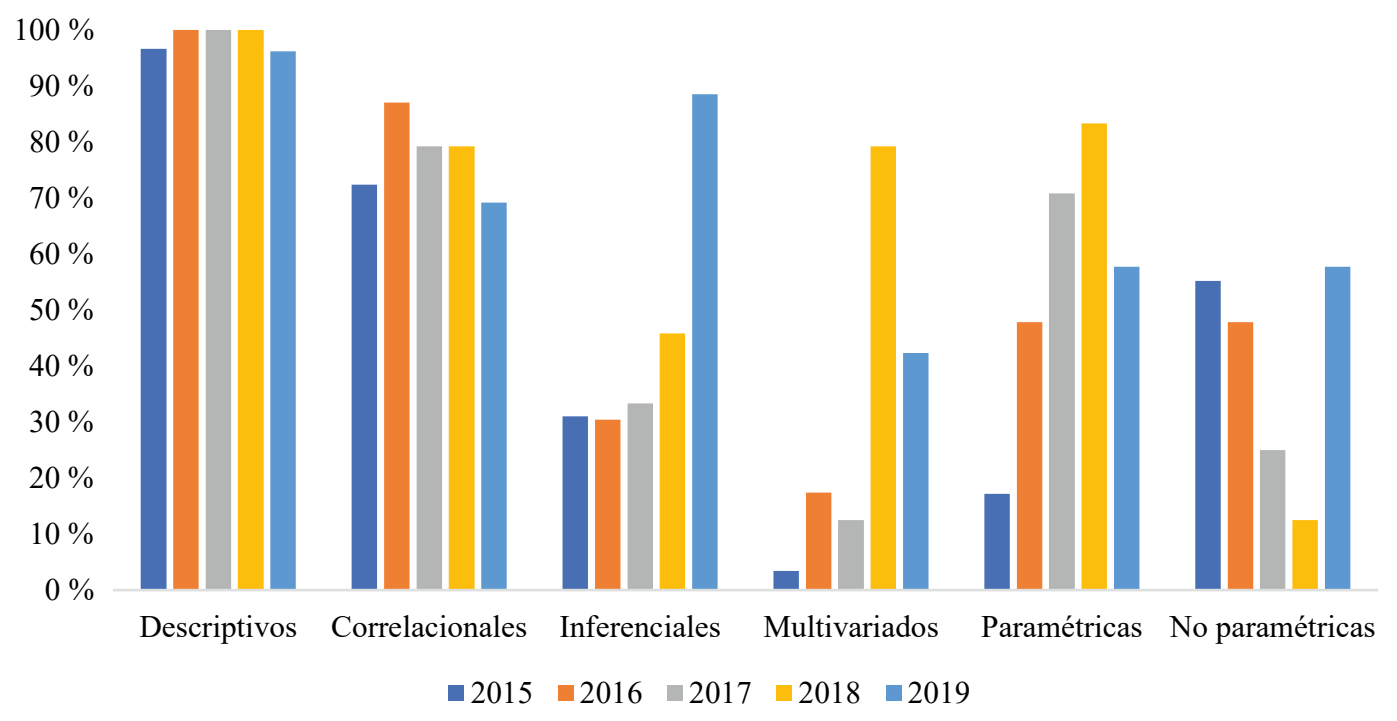

Figura 13. Uso de análisis estadísticos por año.

\section{Cruces por área de conocimiento}

Además de las comparaciones por año, se realizó el análisis por área de conocimiento, en cuyos resultados destaca que prevalece la publicación en español como idioma original del estudio - aunque resalta la publicación en portugués en áreas como bibliometría y adicciones - (véase Figura 14); que en algunas áreas los artículos son escritos por autores principalmente internacionales, con porcentajes superiores al $50 \%$ en algunas de ellas, donde destacan las áreas de psicología social comunitaria, epistemología, psicología clínica y de la salud, adicciones y neuropsicología (véase Figura 15); y que, en cuanto al tipo de muestreo, predomina el uso de muestreo no probabilístico en la mayoría de las áreas, aunque el muestreo probabilístico presenta porcentajes altos en neuropsicología, organizacional y bibliometría (véase Figura 16). También, como se puede observar en la Figura 17, en todas las áreas sobresale el uso de investigación cuantitativa, aunque en las áreas de adicciones, psicología clínica y de la salud, psicología educativa y neuropsicología se emplea la investigación cualitativa o mixta.

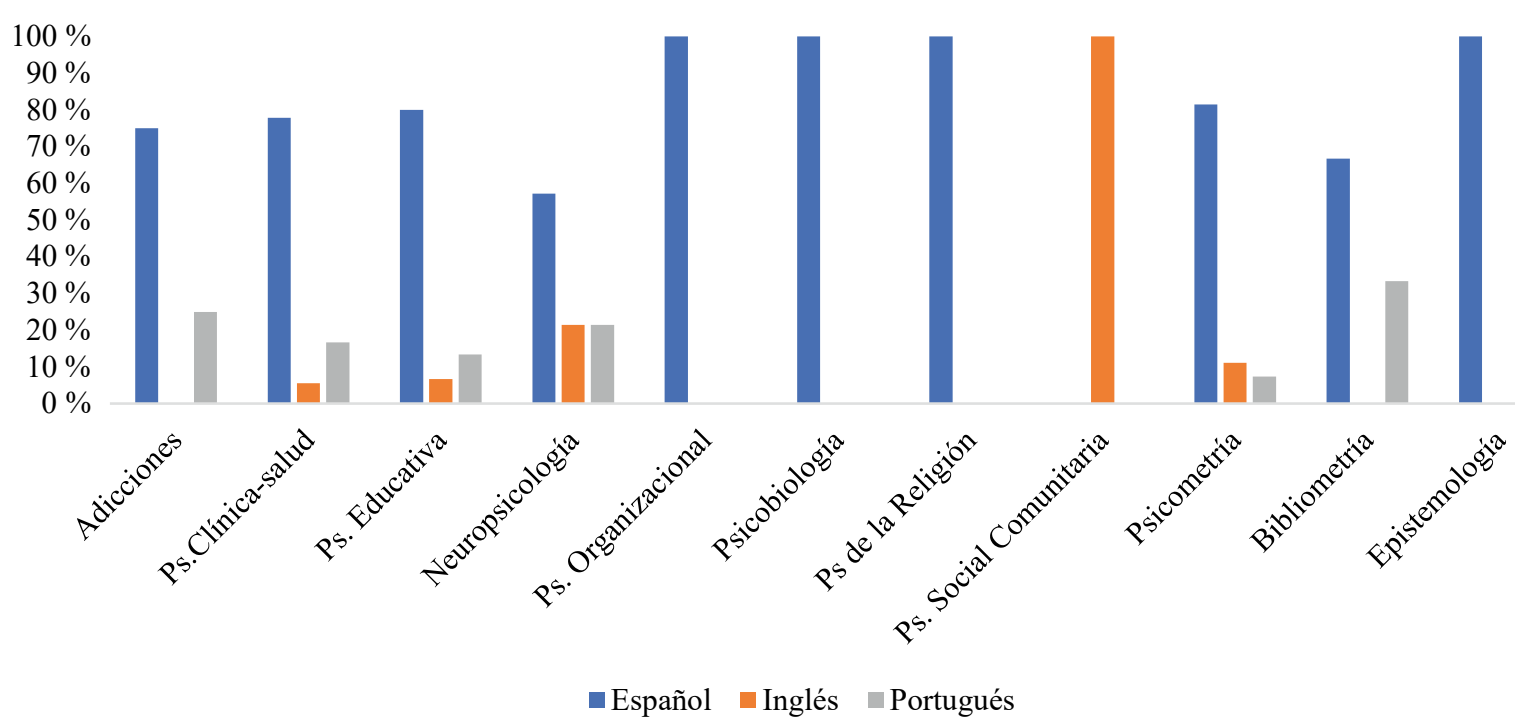

Figura 14. Idioma de publicación según el área de conocimiento. 


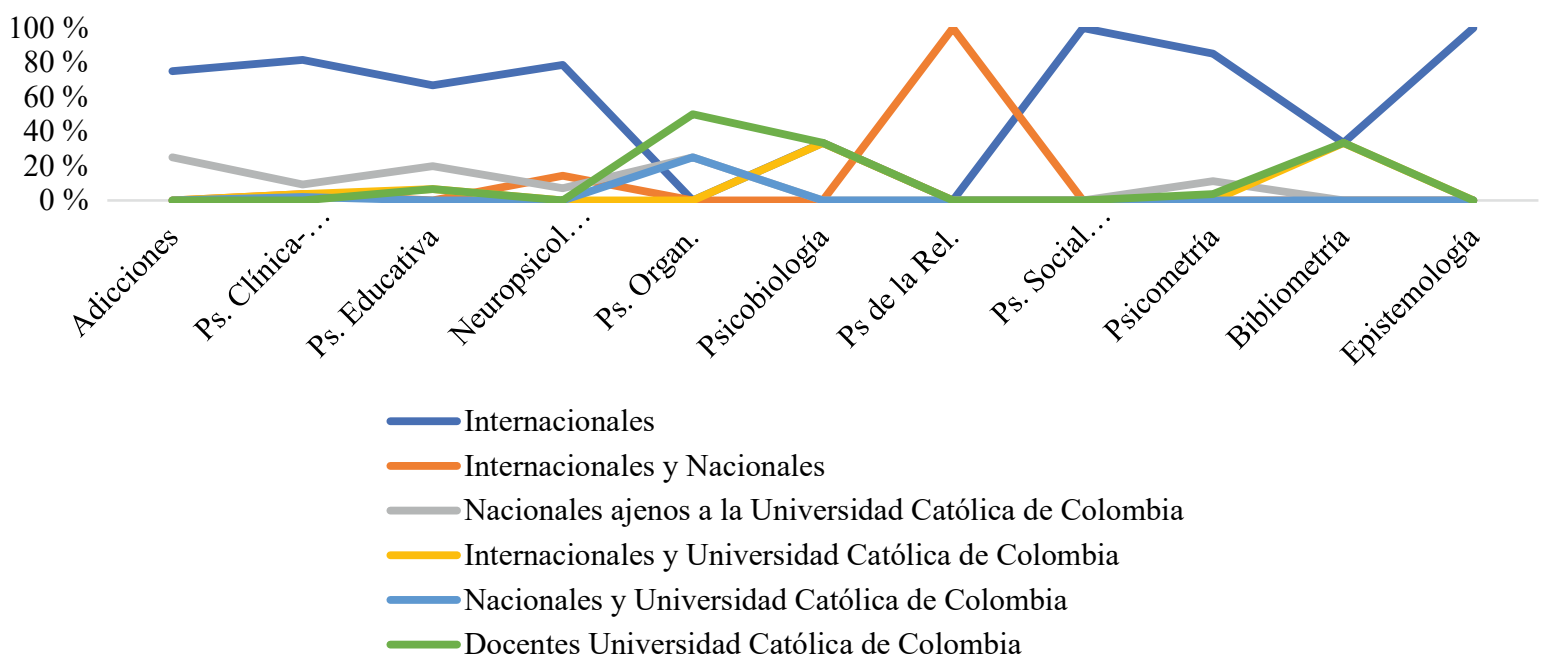

Figura 15. Procedencia de los autores según el área de conocimiento.

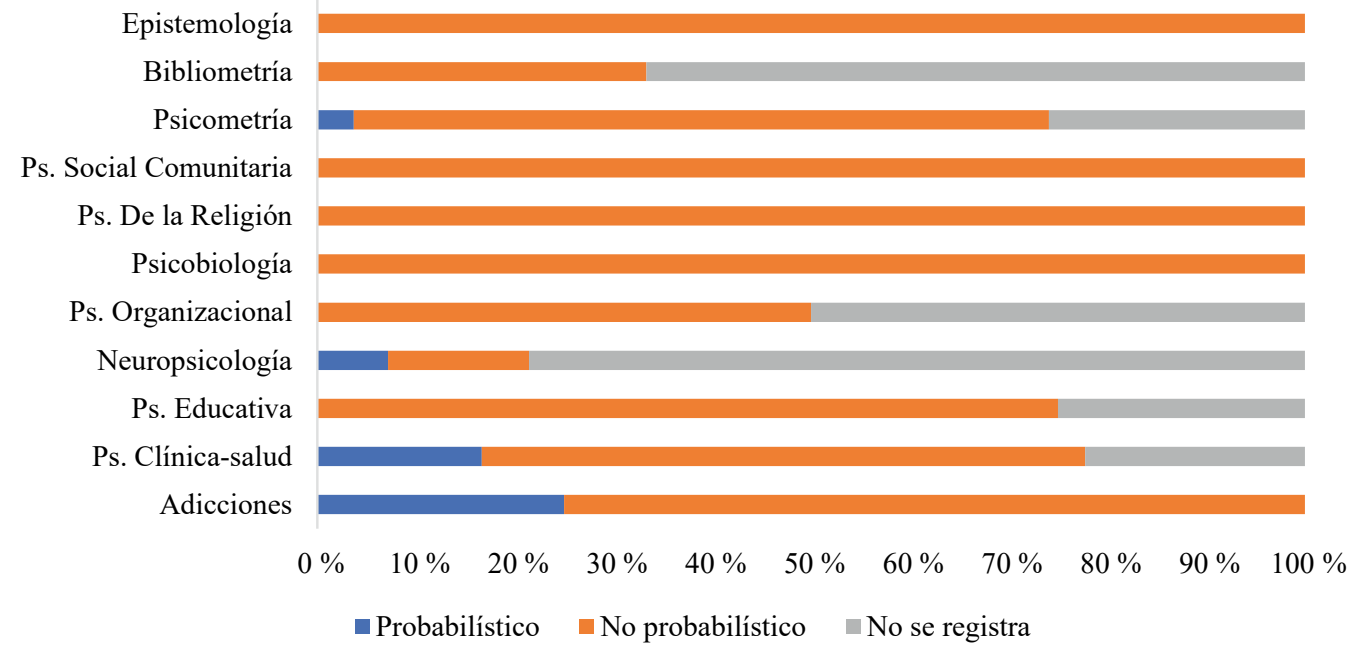

Figura 16. Tipo de muestreo según el área de conocimiento.

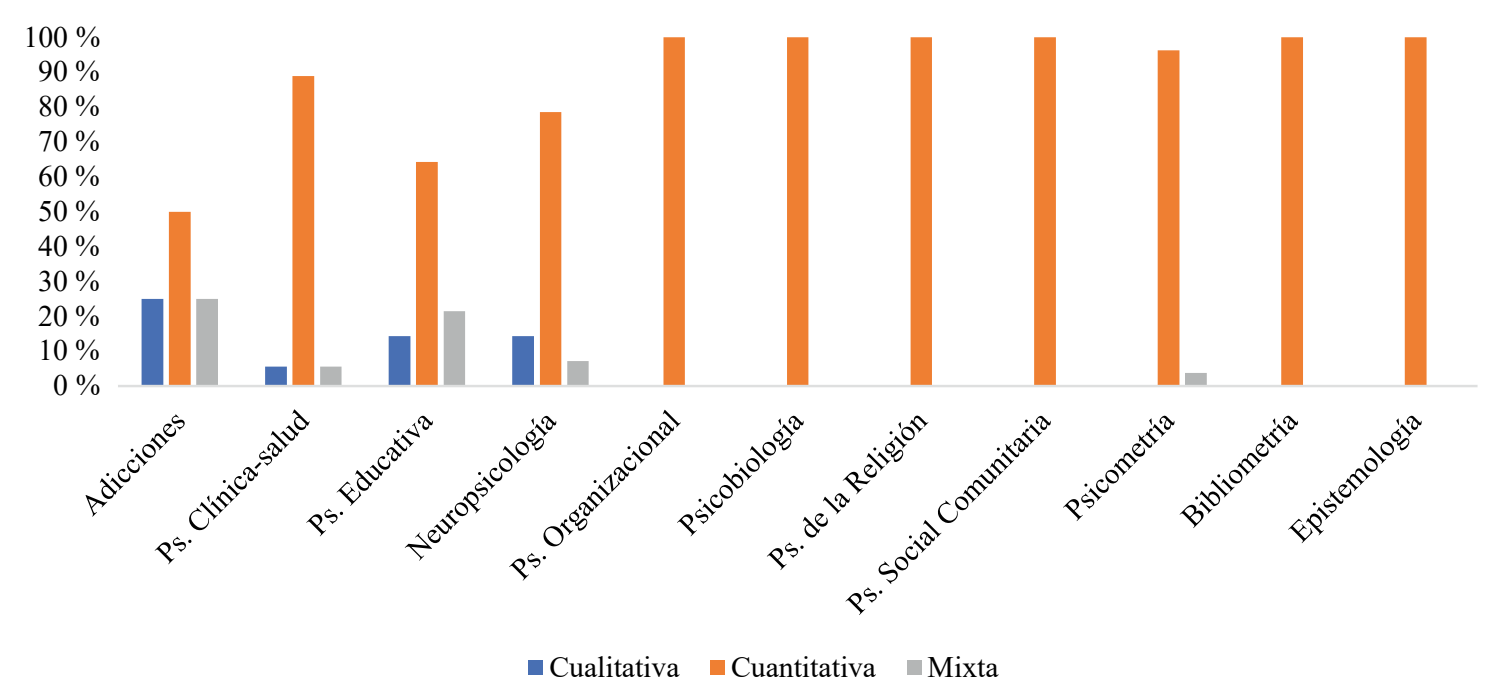

Figura 17. Tipo de investigación según el área de conocimiento. 
Por otra parte, en la Figura 18 se muestra el empleo de los análisis estadísticos en cada una de las áreas, donde se puede encontrar que los análisis descriptivos son usados en todas las áreas, con porcentajes superiores al $90 \%$, y que los análisis correlacionales no son aplicados en las áreas de psicobiología y psicología social comunitaria; además de que se realizan análisis inferenciales en todas las áreas, excepto en psicología de la religión y epistemología, mientras que los análisis multivariados son empleados con porcentajes superiores al $40 \%$ en adicciones, psicobiología y psicometría; también, se encuentra que las pruebas paramétricas fueron aplicadas en todas las áreas, excepto en epistemología, mientras que las no paramétricas no se utilizaron en las áreas de psicología de la religión y bibliometría.

Finalmente, en la Figura 19 se presentan los resultados del promedio de autores por área, donde cabe destacar que las áreas en las que más autores se registran es en neuropsicología y en bibliometría, con 4 y 6 autores en promedio, respectivamente; mientras que el área con menos autores es la organizacional, con estudios realizados por uno o dos autores.

\section{Distribución de las contribuciones}

Con respecto a la distribución por contribuciones, en la Tabla 4 se muestra la distribución de las publicaciones según el sexo del primer autor y el total de autores, donde resalta que en Acta publicó una mayor cantidad de mujeres que de hombres.

Tabla 4

Productividad según el sexo de los autores

\begin{tabular}{ccc}
\hline Sexo & Primer autor $(\%)$ & Total de autores $(\%)$ \\
\hline Hombre & $54(42.52)$ & $176(41.71)$ \\
Mujer & $73(57.48)$ & $247(58.39)$ \\
\hline Total & 127 & 423 \\
\hline
\end{tabular}

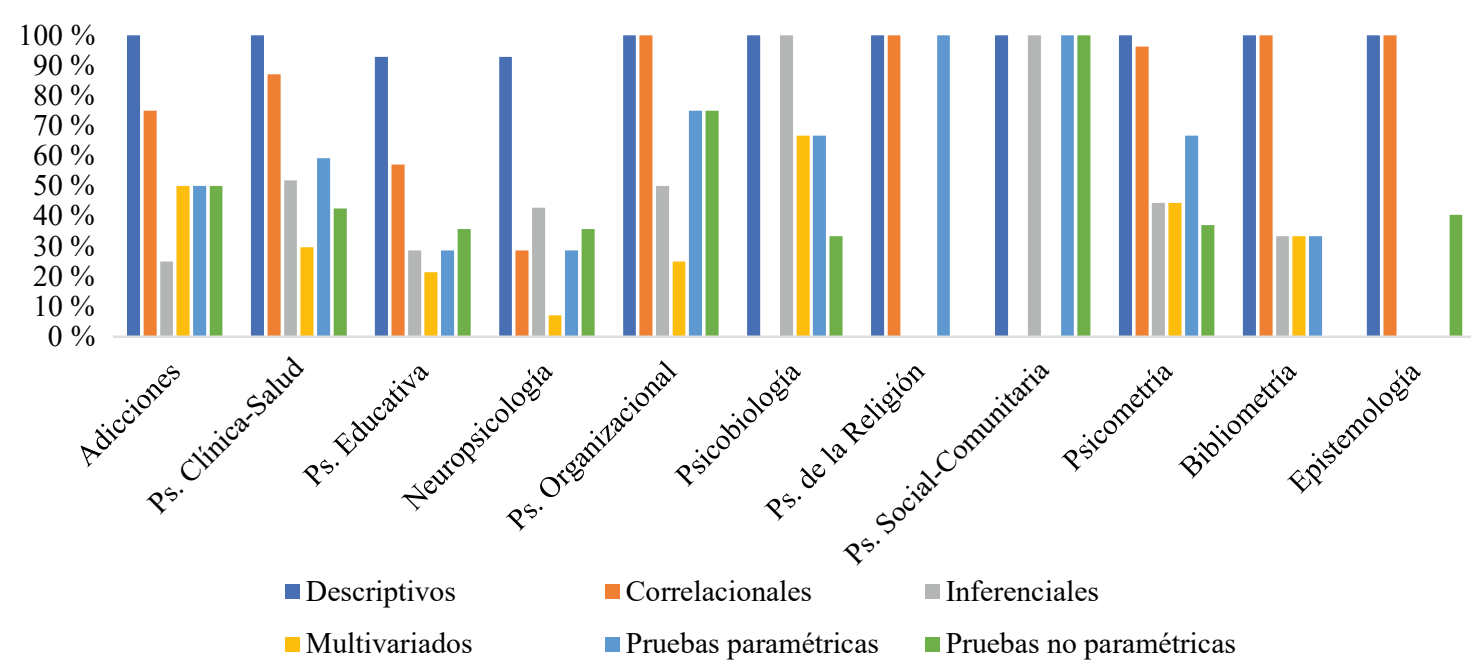

Figura 18. Análisis estadístico empleado según el área de conocimiento.

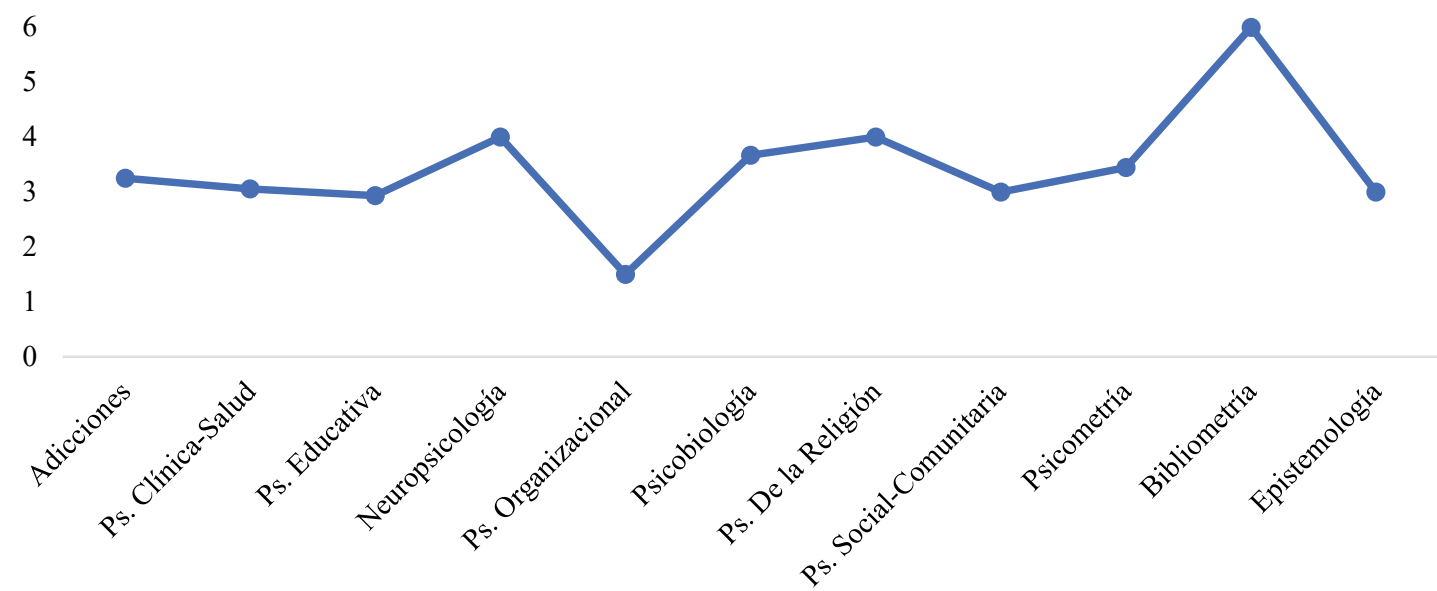

Figura 19. Promedio de autores por área. 
182

Por otra parte, en la Tabla 5 se presenta la cantidad de contribuciones según el autor y el índice de productividad (IP). Para el cálculo del IP se organizaron los datos según el número de contribuciones por autor y se identificó que 371 autores tuvieron una contribución, 23 autores, dos contribuciones, un autor, tres contribuciones, y otro autor, cuatro contribuciones.

Posteriormente, se obtuvo el logaritmo natural del número de contribuciones por autor, cuyo cálculo permitió realizar una clasificación de los autores por niveles de producción. De este modo, los autores se agruparon en: (a) pequeños productores ( $\mathrm{IP}=0)$, (b) medianos productores $(\mathrm{IP}<1)$, y (c) grandes productores (IP $>1)$. Considerando los valores del IP, un total de 371 (93.69\%) autores se ubicaron en el nivel pequeños productores, mientras que $25(6.81 \%)$ autores se encuentran en el nivel medianos productores. No se encontraron grandes productores, lo cual se puede explicar de dos maneras: la primera, por el periodo de análisis, que solo comprendió un rango de cinco años; y segundo, por la política editorial de la revista, en la cual se reglamenta que un autor solo puede publicar en un número de cada volumen.

\section{Colaboración}

Con el fin de analizar la colaboración se calcularon los índices de Lawani y Subramanyam para cada año de publicación. En la Figura 20 se muestra el índice de Lawani, que indica el promedio de autores que firman un artículo, donde se puede observar que estos valores variaron entre 2.83 (2015) y 3.83 (2018), siendo 3.32 el promedio para el periodo 2015-2019. Para la obtención de estos valores se calculó la media ponderada de autores que firmaron los artículos por año y periodo de análisis.

Y, por otra parte, en la Figura 21 se muestra el índice de Subramanyam, en el cual se indica la proporción de autoría múltiple. Allí, en la medida en que el valor se aproxime al uno, se interpreta que se publicó una mayor cantidad de trabajos con autoría múltiple. De este modo, se puede observar que en el periodo 2018 se alcanzó el valor de 1, lo cual permite interpretar que en ese año todos los artículos publicados por Acta tuvieron dos o más autores firmantes. El promedio del periodo 2015-2019 fue de 0.94.

Tabla 5.

Cantidad de contribuciones por autor e índice de productividad

\begin{tabular}{cccc}
\hline Contribuciones por autor & Frecuencia & Porcentaje de contribuciones por autor & Índice de productividad (IP) \\
\hline 1 & 371 & 93.69 & 0 \\
2 & 23 & 5.81 & 0.30 \\
3 & 1 & 0.25 & 0.48 \\
4 & 1 & 0.25 & 0.60 \\
\hline
\end{tabular}

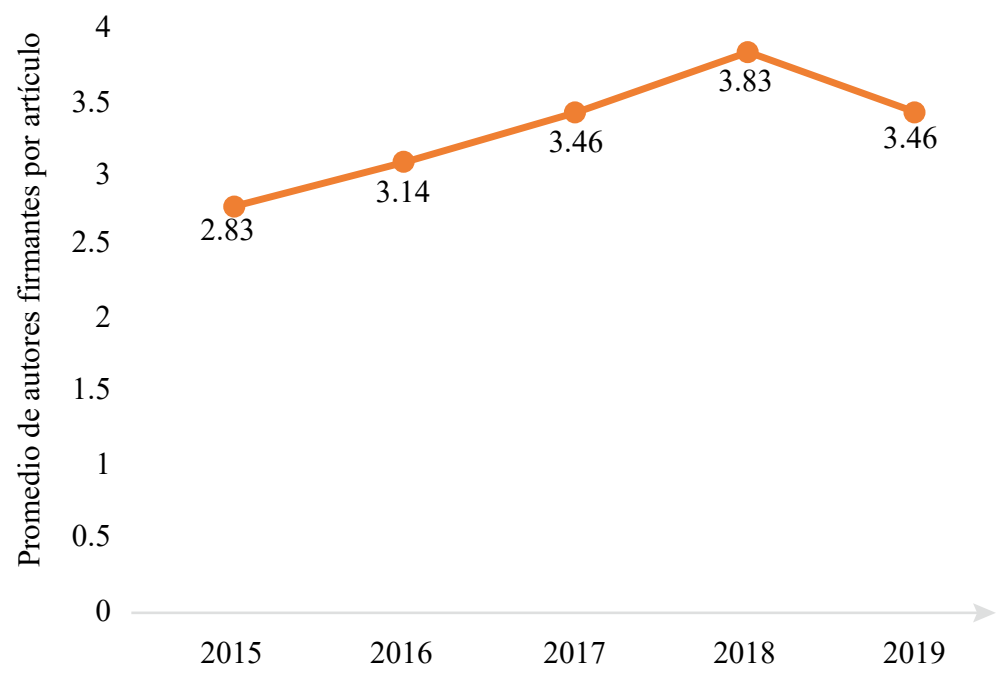

Figura 20. Índice de Lawani. 


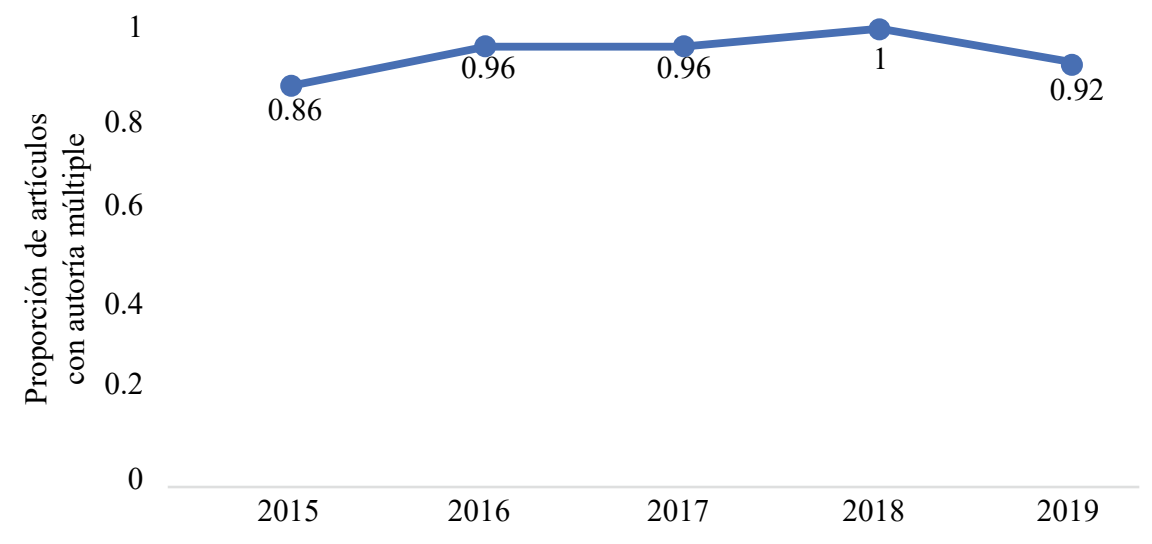

Figura 21. Índice de Subramanyam.

Temáticas y metodologías

Finalmente, en la Tabla 6 se muestra la distribución de los artículos según las áreas temáticas; $y$, con el fin de analizar la concentración temática, se calculó el índice de Pratt para cada año de publicación (véase Figura 22). En este último, se puede observar que estos valores variaron entre 0.44 (2016) y 0.63 (2017), siendo 0.68 el valor para el periodo 20152019. La estimación de estos valores se realizó siguiendo lo propuesto por Gorbea-Portal (2007), a partir de lo cual se fijaron 11 categorías de clasificación temática.

Tabla 6.

Publicaciones por año según el área de conocimiento

\begin{tabular}{ccccccc}
\hline Área & \multicolumn{3}{c}{ Año } & \multirow{2}{*}{ Total (\%) } \\
\cline { 2 - 5 } & 2015 & 2016 & 2017 & 2018 & 2019 & $4(3)$ \\
Adicciones & 2 & 0 & 0 & 2 & 0 & $3(2)$ \\
Bibliometría & 1 & 1 & 0 & 1 & 0 & $54(43)$ \\
Ps. clínica y de la salud & 11 & 8 & 14 & 12 & 9 & $15(14)$ \\
Ps. educativa & 1 & 4 & 3 & 3 & 4 & $1(1)$ \\
Epistemología & 0 & 1 & 0 & 0 & 0 & $14(11)$ \\
Neuropsicología & 8 & 4 & 0 & 1 & 1 & $4(3)$ \\
Ps. organizacional & 1 & 1 & 0 & 0 & 2 & $3(2)$ \\
Psicobiología & 0 & 0 & 1 & 1 & 1 & $27(21)$ \\
Psicometría & 5 & 5 & 5 & 5 & 7 & $1(1)$ \\
Ps. de la religión & 0 & 0 & 1 & 0 & 0 & $1(1)$ \\
Ps. social-comunitaria & 0 & 0 & 0 & 0 & 1 & $1(1)$ \\
\hline
\end{tabular}

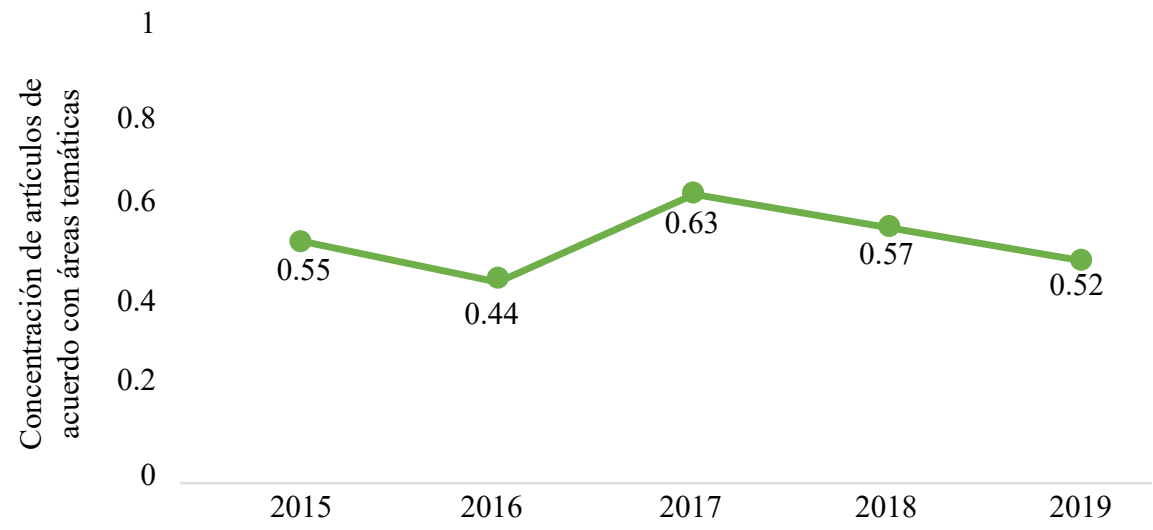

Figura 22. Índice de Pratt. 


\section{Discusión}

El presente estudio corresponde al tercero de una triada de estudios - siendo el primero el de Ravelo et al. (2016), y el segundo el de Salas et al. (2018) — que surgen como respuesta al fortalecimiento de la línea de investigación de estudios bibliométricos a partir de lo publicado en la revista Acta Colombiana de Psicología.

En la ventana del presente estudio, la producción responde a 127 artículos, dos más que los publicados en el periodo 2010-2014. Con respecto a este último período, es importante destacar que el porcentaje de publicaciones a cargo de autores internacionales incrementó en $14.3 \%$ (al pasar de $60.5 \%$ a $74.8 \%$ ); que la producción por áreas mantiene un liderazgo del área de psicología clínica y salud $(38.4 \%$ en el periodo de 2010 a 2014 , y $42.5 \%$ en el periodo de 2015 a 2019); y que en este último periodo se evidencia un incremento en los estudios realizados en el área de psicometría, siendo de $21.3 \%$ de artículos en este quinquenio, comparado con un $12.8 \%$ en el periodo de 2010 a 2014.

Por otra parte, la investigación de corte cuantitativo se mantiene en $87 \%$, la investigación mixta incrementa en un $4.3 \%$, y el uso de instrumentos también incrementa, al pasar del $70.6 \%$ en el periodo de 2010 a 2014 al $96.1 \%$ en el periodo de 2015 a 2019. En cuanto al tipo de muestreo usado para la recolección de información, se encontró una disminución en el porcentaje de publicaciones que no lo registran, al pasar de $43.2 \%$ a $29.1 \%$, además de que se siguen utilizando con un alto porcentaje los análisis descriptivos $(97.6 \%)$, así como los estadísticos correlacionales, que pasaron de un porcentaje de uso del $38.6 \%$ al $76.4 \%$.

Asimismo, el promedio de autores por artículo pasó de 2.64 a 3.32 de un quinquenio al otro, y el promedio del número de referencias incrementó en seis, al pasar de 39 a 45. Asimismo, se destaca el tiempo transcurrido entre el recibido del documento y su aceptación, que disminuyó en 3.18 meses, lo cual muestra el acertado y estratégico manejo por parte del equipo editorial de la revista en relación con los tiempos dedicados para el proceso de arbitraje.

De otra parte, los índices de colaboración empleados en el presente estudio evidencian un incremento importante, cuestión que se potencia con el acceso abierto (GabrielleBreugelmans et al., 2018) y que se asocia a un alza de las citas en el caso de la colaboración internacional (Gonçalves et al., 2019). La concentración temática de la revista se aproxima a un nivel medio, aspecto que es importante mantener.

A pesar de que Acta es una revista generalista (miscelánea), es decir, abierta a una diversidad de tópicos en psicología, tras el presente análisis bibliométrico se puede observar que existe una inclinación natural por la psicología clínica, lo que podría obedecer a la "historia intelectual" propia de la revista, constructo utilizado para significar una suerte de coherencia teórica existente en las publicaciones desde su fundación (Comins \& Leydesdorff, 2016). Salvaguardar la tradición temática de una revista no es una cuestión baladí, sino que se torna cada vez más difícil cuando se trata de cumplir con criterios de internacionalización e impacto en sistemas de información de alta calidad (Navarrete-Cortes et al., 2010).

Respecto a esto último, Acta ha cumplido con creces en el tema de internacionalización de la mayor parte de los autores de sus contribuciones, a la vez que ha logrado su inclusión en el Emerging Sources Citation Index (ESCI) de Web of Science, lo cual constituye una fortaleza para ganar visibilidad en el mundo científico y ampliar la cobertura geográfica en relación con la recepción de artículos.

Adicional a lo anterior, es de gran importancia resaltar las buenas prácticas editoriales que tiene la revista, entre las cuales destacan la decisión de traducir al inglés todos los artículos, tener un buen promedio en los tiempos entre recepción y envío de dictamen, así como evitar caer en ciertas lógicas de la producción masiva, como el fenómeno conocido como hiperautoría, es decir, la publicación de artículos con un número desproporcionado de coautores, generalmente más de 35 (Ahmed et al., 1997; Cronin, 2001).

Finalmente, los desafíos que tiene Acta, y en general las revistas latinoamericanas en la actualidad, tienen que ver, esencialmente, con buscar cada vez más cooperación entre revistas latinoamericanas, así como establecer redes que conlleven al fortalecimiento de la infraestructura editorial, la visibilización y el impacto en citas (López-López, 2019). Además, también resulta de gran importancia destacar que tanto Acta como otras revistas de psicología latinoamericanas son proyectos que tienen el enorme reto de reinventarse y transformarse continuamente, sobre todo con el propósito de hacerle frente tanto a la asimetría existente entre las revistas latinoamericanas con respecto a la producción internacional (Jaraba-Barrios et al., 2012), como a algunos desaciertos de la política de evaluación de las revistas científicas (Publindex) en Colombia (López-López, 2019).

\section{Referencias}

Aguado-López, E., López-López, W., Becerril-García, A., \& Salas, G. (2017). Patrones de internacionalización en psicología desde la Revista Interamericana de Psicología. Interamerican Journal of Psychology, 51(3), 268-281. https://doi.org/10.30849/rip/ijp.v51i3.897 
Ahmed, S. M., Maurana, C. A., Engle, J. A., Uddin, D. E., \& Glaus, K. D. (1997). A method for assigning authorship in multiauthored publications. Family Medicine, 29(1), 42-44. https://www.ncbi.nlm.nih.gov/pubmed/9007560

Alhuay-Quispe, J., \& Pacheco-Mendoza, J. (2018). Escaso uso de indicadores de productividad científica en estudios bibliométricos. Educación Médica, 19(2), 128-130. https:// doi.org/10.1016/j.edumed.2017.04.013

Ardila, R. (1999). Las ideas psicológicas en Colombia. Revista de Estudios Sociales, 3, 59-67. https://doi.org/10.7440/ res3.1999.04

Ardila, R. (2006). Editorial. Acta Colombiana de Psicología, 9(1), 3-4. https://actacolombianapsicologia.ucatolica.edu. co/article/view/408

Ardila, R., \& Pérez-Acosta, A. M. (2010). La psicología colombiana: revistas y bibliografía. Revista Latinoamericana de Psicología, 42(3), 517-523. https://doi.org/10.15446/rcp

Bonnevie, E. (2002). A multifaceted portrait of a library and information science journal: the case of the Journal of Information Science. Journal of Information Science, 29(1), 11-23. https://doi.org/10.1177/016555150302900102

Calver, M. C., Lilith, M., \& Dickman, C. R. (2013). A 'perverse incentive' from bibliometrics: could National Research Assessment Exercises (NRAEs) restrict literature availability for nature conservation? Scientometrics, 95(1), 243255. https://doi.org/10.1007/s11192-012-0908-1

Comins, J. A., \& Leydesdorff, L. (2016). RPYS i/o: software demonstration of a web-based tool for the historiography and visualization of citation classics, sleeping beauties and research fronts. Scientometrics, 107(3), 1509-1517. https:// doi.org/10.1007/s11192-016-1928-z

Cronin, B. (2001). Hyperauthorship: A postmodern perversion or evidence of a structural shift in scholarly communication practices? Journal of the American Society for Information Science and Technology, 52(7), 558-569. https://doi. org/10.1002/asi.1097

Cruz-Becerra, D. (2003). Editorial. Acta Colombiana de Psicología, 10, 3-6. https://actacolombianapsicologia.uca tolica.edu.co/article/view/491/488

Cudina, J. N., Millán, J. D., \& Ossa, J. C. (2017). Redes de comunicación científica en la investigación psicológica de las Américas a través de la revista Interamericana de psicología. Interamerican Journal of Psychology, 51(3), 282-296. https://doi.org/10.30849/rip/ijp.v51i3.898

Daniel, H. A. (2005). Publications as a measure of scientific advancement and of "scientist's productivity. Learned publishing, 18(2), 143-148. https://doi. org/10.1087/0953151053584939

Egghe, L. (1988). On the classification of the classical bibliometric laws. Journal of Documentation, 44(1), 53-62. https://doi.org/10.1108/eb026819

Fierro, C., Brisuela, L., Bruna, O., \& Biglieri, F. (2017). ¿Pluralismo o monoteoría en psicología clínica?
Análisis empírico de problemas epistemológicos y formativos en carreras de psicología en Argentina (2015). Revista Guillermo de Ockham, 15(2), 51-68. https://doi. org/10.21500/22563202.3294

Flórez-Alarcón, L. (1998). Centro de Estudios e investigaciones sobre adicciones. Acta Colombiana de Psicología, 1, 121-124. https://actacolombianapsicologia.ucatolica.edu. co/article/view/636

Flórez-Alarcón, L. (2000). Editorial Revista Acta Colombiana de Psicología. Acta Colombiana de Psicología, 4, 3-6. https://actacolombianapsicologia.ucatolica.edu.co/article/ view $/ 596$

Gabrielle-Breugelmans, J., Roberge, G., Tippett, C., Durning, M., Struck, D. B., \& Makanga, M. M. (2018). Scientific impact increases when researchers publish in open access and international collaboration: A bibliometric analysis on po verty-related disease papers. PLOS ONE, 13(9), e0203156. https://doi.org/0.1371/journal.pone.0203156

Gómez-Morales, Y. J., Jaraba-Barrios, B., Guerrero-Castro, J., \& López-López, W. (2012). Entre Internacionalización y Consolidación de Comunidades Académicas Locales. Sobre la Revista Latinoamericana de Psicología. Revista Colombiana de Psicología, 21(1), 97-110. https://doi. org/10.15446/rcp

Gonçalves, A. P., Porto, B. L., Rodolfo, B., Faggion, C. M., Jr., Agostini, B. A., Sousa-Neto, M. D., \& Moraes, R. R. (2019). Brazilian articles in top-tier dental journals and influence of international collaboration on citation rates. Brazilian Dental Journal, 30(4), 307-316. https://doi. org/10.1590/0103-6440201902826

Gorbea-Portal, S. (2007). Principales revistas latinoamericanas en ciencias bibliotecológicas y de la información: su difusión y su concentración temática y geográfica. Investigación Bibliotecológica, 21(42), 79-108. https://doi.org/10.22201/ iibi.0187358xp.2007.42.4119

Hernández-Pozo, M. (2013). Una mirada a la investigación actual en Latinoamérica sobre equidad y estudios de género. Acta Colombiana de Psicología, 16(2), 11-14. https://acta colombianapsicologia.ucatolica.edu.co/article/view/175

Huang, L., Zhang, Y., Guo, Y., Zhu, D., \& Porter, A. (2014). Four dimensional Science and Technology planning: A new approach based on bibliometrics and technology roadmapping. Technological Forecasting and Social Change, 8, 3948. https://doi.org/10.1016/j.techfore.2012.09.010

Jaraba-Barrios, B., Guerrero-Castro, J., Gómez-Morales, Y., \& López-López, W. (2012). Bibliometría e historia de las prácticas académicas locales: un esbozo a partir del caso de la psicología en Colombia. Avances en Psicología Latinoamericana, 29(2), 354-369. https://revistas.urosario. edu.co/index.php/apl/article/view/2008

Kamdem, J. P., Duarte, A. E., Rodrigues-Lima, K. R., BatistaTexeira, J. B., Waseem-Hassan, R., ... Tsopmo, A. (2019). Research trends in food chemistry: A bibliometric review of 
its 40 years anniversary (1976-2016). Food Chemistry, 294, 448-457. https://doi.org/10.1016/j.foodchem.2019.05.021

Lawani, S. M. (1981). Bibliometrics: Its Theoretical Foundations, Methods and Applications. Libri, 31(1), 294315. https://doi.org/10.1515/libr.1981.31.1.294

Lawani, S. M. (1986). Some bibliometric correlates of quality in scientific research. Scientometrics, 9(1-2), 13-25. https:// doi.org/10.1007/BF02016604

Leydesdorff, L. (2007). Scientific communication and cognitive codification. Social system theory and the sociology of scientific knowledge. European Journal of Social Theory, 10(3), 375-388. https://doi.org/10.1177/1368431007080701

Lievrouw, L. A. (1989). The Invisible College Reconsidered: Bibliometrics and the Development of Scientific Communication Theory. Communication Research, 16(5), 615-628. https://doi.org/10.1177/009365089016005004

López-López, W. (2019). ¿Qué es un editor de una revista científica en América Latina? Universitas Psychologica, 18(3), 1-4. https://doi.org/10.11144/Javeriana.upsy18-3.qerc

López-López, W., \& Calvache, O. (1998). La psicología de habla hispana: 30 años de la Revista Latinoamericana de psicología. Revista Latinoamericana de Psicología, 30(3), 401427. https://www.redalyc.org/articulo.oa?id=805/80530302

Lozano-Ardila, M. C. (2000). Editorial Revista Acta Colombiana de Psicología. Acta Colombiana de Psicología, 2, 3-7. https://actacolombianapsicologia.ucatolica.edu.co/ article/view/607

Milfont, T. L., Amirbagheri, K., Hermanns, E., \& Merigó, J. M. (2019). Celebrating Half a Century of Environment and Behavior: A Bibliometric Review. Environment and Behavior, 51(5), 469-501. https://doi. org/10.1177/0013916519843126

Millán, J. D., Polanco, F., Ossa, J. C., Béria, J., \& Cudina, J. N. (2017). La cienciometría, su método y su filosofía: Reflexiones epistémicas de sus alcances en el siglo XXI. Revista Guillermo de Ockham, 15(2), 17-27. https://doi. org/10.21500/22563202.3492

Molano-García, B. (1999). Editorial Revista Acta Colombiana de Psicología. Acta Colombiana de Psicología, 2, 3-9. https://actacolombianapsicologia.ucatolica.edu.co/article/ view/616

Montero, I., \& León, O. G. (2007). A guide for naming research studies in psychology. International Journal of Clinical and Health Psychology, 7, 847-862. https://www.redalyc.org/ pdf/337/33770318.pdf

Morgado-Gallardo, K., Salas, G., Faúndez, M. J., López-López, W., Ventura-León, J., Barboza-Palomino, M., ... GuerraLabbé, L. (2018). 25 años de Suma Psicológica. Un análisis bibliométrico. Suma Psicológica, 25(2), 90-101. https://doi. org/10.14349/sumapsi.2018.v25.n2.1.

Navarrete-Cortes, J., Fernández-López, J. A., López-Baena, A., Quevedo-Blasco, R., \& Buela-Casal, G. (2010). Global Psychology: a bibliometric analysis of Web of Science
Publications. Universitas Psychologica, 9(2), 553-567. https://doi.org/10.11144/Javeriana.upsy9-2.gpba

Pratt, A. D. (1977). A measure of class concentration in bibliometrics. Journal of the American Society for Information Science, 28(5), 285-292. https://doi.org/10.1002/ asi.4630280508

Pérez-Acosta, A. (2002). Editorial Revista Acta Colombiana de Psicología. El análisis del comportamiento humano en hispanoamérica. Acta Colombiana de Psicología, 8, 3-5. https://actacolombianapsicologia.ucatolica.edu.co/article/ view/1953

Puche-Navarro, R., \& Ossa, J. C. (2012). Claves de la Publicación Psicológica en Colombia: ritmo, grupos y modalidades de producción en la práctica investigativa. Revista colombiana de psicología, 21(1), 79-95. https://doi. org $/ 10.15446 / \mathrm{rcp}$

Ravelo-Contreras, E. L. (2007). Editorial. Acta Colombiana de Psicología, 10(1), 3-4. https://actacolombianapsicologia. ucatolica.edu.co/article/view/225

Ravelo-Contreras, E. L. (2008). Editorial. Acta Colombiana de Psicología, 11(1), 7-8. https://actacolombianapsicologia. ucatolica.edu.co/article/view/310

Ravelo-Contreras, E. L. (2016). Editorial. Acta Colombiana de Psicología, 19(1), 7-8. https://doi.org/10.14718/ ACP.2016.19.1.1

Ravelo-Contreras, E. L. (2017). Editorial. Acta Colombiana de Psicología, 20(1), 7. https://doi.org/10.14718/ ACP.2017.20.1.1

Ravelo-Contreras, E. L., Mejía, S., \& González, M. E. (2016). La producción científica de acta colombiana de psicología: análisis descriptivo del periodo 2010-2014. Acta Colombiana de Psicología, 19(2), 281-297. https://doi. org/10.14718/ACP.2016.19.2.12

Salas, G., Ponce, F. P., Méndez-Bustos, P., Vega-Arce, M., Pérez, M., López-López, W., \& Cárcamo-Vásquez, H. (2017). 25 Años de Psykhe: Un Análisis Bibliométrico. Psykhe, 26(1), 1-17.

Salas, G., Ravelo-Contreras, E. L., Mejía, S., Andrades, R., Acuña, E., ... Pérez-Acosta, A. M. (2018). Dos décadas de Acta Colombiana de Psicología: un análisis bibliométrico. Acta Colombiana de Psicología, 21(2), 13-38. https://doi. org/10.7764/psykhe.26.1.1205

Salas, G., Vega-Arce, M., González, C., Ossa, J. C., Cudina, J. N., ... López-López, W. (2019). The Fiftieth Anniversary of the Revista Latinoamericana de Psicología. Revista Latinoamericana de Psicología, 51(2), 206-218. https://doi. org/10.14349/rlp.2019.v.51.n2.7

Salvador-Cruz, J. (2014). Editorial. Acta Colombiana de Psicología, 17(2), 7-8. https://actacolombianapsicologia. ucatolica.edu.co/article/view/15

Sigué, S. P. (2019). In Celebration of the 20 th Anniversary of Journal of African Business. Journal of African Business, 
20(2), 155-159. https://doi.org/10.1080/15228916.2019.16 07421

Solís-Cámara, P., \& Díaz-Romero, M. (2007). Editorial. Acta Colombiana de Psicología, 10(2), 5-6. https://actacolom bianapsicologia.ucatolica.edu.co/article/view/206

Subramanyan, K. (1983). Bibliometric studies of research collaboration: a review. Journal of Information Science, 6, 3338. https://doi.org/10.1177/016555158300600105

Travis-Nicholls, P. (1989). Bibliometric modeling processes and the empirical validity of Lotka's Law. Journal of the American Society for Information Science, 40(6), 379-385. https://doi.org/10.1002/(SICI)10974571(198911)40:6<379::AID-ASI1>3.0.CO;2-Q

Tsay, M. (2003). Literature growth, journal characteristics, and author productivity in subject indexing, 1977 to 2000. Journal of the American Society for information Science and Technology, 55(1), 64-73. https://doi.org/10.1002/ asi. 10346

Tsay, M.Y., Jou, S. J., \& Ma, S. S. (2000). A Bibliometric study of semiconductor literature, 1978-1997. Scientometrics, 49(3), 491-509. https://doi.org/10.1023/A:1010593824716

Universidad Católica de Colombia. (2015-2019). Portal de revistas: Acta Colombiana de Psicología. https://editorial. ucatolica.edu.co/index.php/acta-colombiana-psicologia/
Utap-Anyi, K., Zainab, A. N., \& Anuar, N. B. (2009). Bibliometrics studies on single journals: a review. Malaysian Journal of Library \& Information Science, 14(1), 17-55. https://ejournal.um.edu.myindex.php/MJLIS/ article/view/6951

Vargas-Espinosa, N. M. (2001). Editorial Revista Acta Colombiana de Psicología. Acta Colombiana de Psicología, 6, 3-8. https://actacolombianapsicologia.ucatolica.edu.co/ article/view/577

Vargas-Ordoñez, C. (1998). Editorial Revista Acta Colombiana de Psicología. Acta Colombiana de Psicología, 1, 5-6. https://actacolombianapsicologia.ucatolica.edu.co/article/ view/627

Vinkler, P. (2010). Indicators are the essence of scientometrics and bibliometrics. Scientometrics, 85(3), 861-866. https:// doi.org/10.1007/s11192-010-0159-y

Wang, C., Lim, M. K., \& Lyons, A. (2019). Twenty years of the International Journal of Logistics Research and Applications: a bibliometric overview. International Journal of Logistics Research and Applications, 22(3), 304323. https://doi.org/10.1080/13675567.2018.1526262 